\title{
Scan-plane truncation in diffraction tomography-based imaging
}

\author{
Rubæk, Tonny; Meincke, Peter
}

Published in:

IEEE AP-S International Symposium

Link to article, DOI:

10.1109/APS.2005.1551517

Publication date:

2005

Document Version

Publisher's PDF, also known as Version of record

Link back to DTU Orbit

Citation (APA):

Rubæk, T., \& Meincke, P. (2005). Scan-plane truncation in diffraction tomography-based imaging. In IEEE AP-S International Symposium (Vol. 1B, pp. 187-189). IEEE. https://doi.org/10.1109/APS.2005.1551517

\section{General rights}

Copyright and moral rights for the publications made accessible in the public portal are retained by the authors and/or other copyright owners and it is a condition of accessing publications that users recognise and abide by the legal requirements associated with these rights.

- Users may download and print one copy of any publication from the public portal for the purpose of private study or research.

- You may not further distribute the material or use it for any profit-making activity or commercial gain

- You may freely distribute the URL identifying the publication in the public portal

If you believe that this document breaches copyright please contact us providing details, and we will remove access to the work immediately and investigate your claim 


\title{
Scan-Plane Truncation in Diffraction Tomography-Based Imaging
}

\author{
Tonny Rubæk* and Peter Meincke \\ Ørsted·DTU, Technical University of Denmark \\ \{tru,pme\}@oersted.dtu.dk
}

Three approaches for truncating the scan plane in diffraction tomography-based imaging are suggested and compared. The first and second approaches involve multiplication of a window to the scattered field before evaluating the spatial Fourier transform. The third method relies on an asymptotic end-point expansion of the Fourier integral.

\section{Introduction}

The spatial Fourier transform of the scattered field on a planar scan plane occurs in diffraction tomography-based imaging derived from Devaney's filtered-backpropagation algorithm $[1,2]$. To avoid aliasing problems, the scan plane must be large enough to intercept all energy of the scattered field. However, in many practical configurations it is impossible to realize the scan plane size as dictated by this requirement, and a truncated scan plane must therefore by applied. The straight-forward approach is simply to calculate the Fourier transform over the truncated scan plane, corresponding to applying a rectangular window, but this method does not eliminate aliasing. Instead, a window possessing small side lobes in the Fourier domain - as for instance the Blackman-Harris window [3] is preferred. As an alternative to using windows, the truncation of the scan plane can be accounted for by employing an asymptotic end-point contribution of the Fourier integral.

\section{The Formulation}

The considered measurement configuration is a microwave camera consisting of a transmitting and receiving antenna, operating in fixed offset. The scan is performed with the receiving antenna in the $z=0$ plane, and the positions of the receiving antenna and the offset are given by

$$
\boldsymbol{r}_{\boldsymbol{r}}=x_{r} \hat{\boldsymbol{x}}+y_{r} \hat{\boldsymbol{y}} \quad \text { and } \quad \boldsymbol{r}_{\boldsymbol{\Delta}}=x_{\Delta} \hat{\boldsymbol{x}}+y_{\Delta} \hat{\boldsymbol{y}}+z_{\Delta} \hat{\boldsymbol{z}}
$$

respectively. Both antennas are assumed to be Hertzian dipoles with dipole moments $\hat{\boldsymbol{p}}_{\boldsymbol{r}}$ and $\hat{\boldsymbol{p}}_{\boldsymbol{t}}$ for the receiving and transmitting antennas, respectively. The microwave camera is operating in the frequency range given by the minimum angular frequency $\omega_{\min }$ and the maximum angular frequency $\omega_{\max }$. The scattering objects are positioned in the lower half-space $(z<0)$ and both the scattering objects and the microwave camera are positioned in free space. The non-conducting scattering objects are assumed to have a permittivity $\Delta \epsilon(\boldsymbol{r})$ slightly different than that of free space, so that the scattering process can be accurately described by the first-order Born approximation. By application of this approximation, the forward model and imaging algorithm in [4] can be applied, using two identical background media. Using the time factor $e^{-i \omega t}$, the imaging algorithm is given by

$$
\begin{aligned}
\Delta \epsilon(\boldsymbol{r}) & =\Re\left\{\frac{64 z}{\pi^{2} \mu_{0}^{2} c_{1}^{3}} \int_{\omega_{\min }}^{\omega_{\max }} \iint_{k_{x}^{2}+k_{y}^{2}<4 k_{1}^{2}} \hat{\boldsymbol{p}}_{\boldsymbol{r}} \cdot \widetilde{\boldsymbol{E}_{\boldsymbol{s}}}\left(k_{x}, k_{y}, \omega\right) e^{i\left[k_{x}\left(x-\frac{1}{2} x_{\Delta}\right)+k_{y}\left(y-\frac{1}{2} y_{\Delta}\right)\right]}\right. \\
& \left.\cdot \frac{e^{i\left[z \sqrt{4 k_{1}^{2}-k_{x}^{2}-k_{y}^{2}}-\frac{1}{2} \Delta z \sqrt{4 k_{0}^{2}-k_{x}^{2}-k_{y}^{2}}\right]}}{\omega\left(4 k_{1}^{2}-k_{x}^{2}-k_{y}^{2}\right)^{\frac{3}{2}} I(\omega) \hat{\boldsymbol{p}}_{\boldsymbol{r}} \cdot \overline{\overline{\boldsymbol{F}}}\left(k_{x}, k_{y}, \omega\right) \cdot\left[\hat{\boldsymbol{p}}_{\boldsymbol{t}} \cdot \overline{\overline{\boldsymbol{F}}}\left(k_{x}, k_{y}, \omega\right)\right]} \mathrm{d} k_{y} \mathrm{~d} k_{x} \mathrm{~d} \omega\right\} .
\end{aligned}
$$


Herein, the dyadic $\overline{\overline{\boldsymbol{F}}}$ is the plane-wave expansion of the dyadic Green's function for observation point and source point above and below the $z=0$ plane, respectively [4, Eq. 6]. The quantity $\widetilde{\boldsymbol{E}_{\boldsymbol{s}}}$ is the two-dimensional Fourier transform of the scattered field, defined as

$$
\widetilde{\boldsymbol{E}_{\boldsymbol{s}}}\left(k_{x}, k_{y}, \omega\right)=\int_{-\infty}^{\infty} \int_{-\infty}^{\infty} \boldsymbol{E}_{\boldsymbol{s}}\left(\boldsymbol{r}_{\boldsymbol{r}}, \omega\right) e^{-i\left[k_{x} x_{r}+k_{y} y_{r}\right]} \mathrm{d} x_{r} \mathrm{~d} y_{r} .
$$

Since the integration in this expression is to be performed over the entire infinite plane $z=z_{r}$, and the scattered field is only measured in the finite scan plane given by

$$
\left|x_{r}\right| \leq x_{0} \quad \text { and } \quad\left|y_{r}\right| \leq y_{0},
$$

only an approximate Fourier transform can be calculated. This will result in a distortion of the calculated image. It is therefore of interest to investigate how the truncation of the scan plane influences the Fourier transform.

\section{Scan Plane Truncation}

Three approaches for truncating the scan plane have been investigated. The first is a simple truncation in which the field outside the scan plane is assumed to be zero. This corresponds to limiting the scan plane by using a simple rectangular window and the resulting expression for the Fourier integral is given as

$$
\widetilde{\boldsymbol{E}_{\boldsymbol{s}}^{\text {rect }}}\left(k_{x}, k_{y}, \omega\right)=\int_{-y_{0}}^{y_{0}} \int_{-x_{0}}^{x_{0}} \boldsymbol{E}_{\boldsymbol{s}}\left(\boldsymbol{r}_{\boldsymbol{r}}, \omega\right) e^{-i\left[k_{x} x_{r}+k_{\boldsymbol{y}} y_{r}\right]} \mathrm{d} x_{r} \mathrm{~d} y_{r} .
$$

Since the use of the rectangular window is known to give rise to aliasing, the effect of using a more advanced window has also been tested. In this paper the Blackman-Harris window [3] has been applied and the resulting expression for the Fourier transform of the scattered field is

$$
\widetilde{\boldsymbol{E}_{\boldsymbol{s}}^{\text {B.H. }}}\left(k_{x}, k_{y}, \omega\right)=\int_{-y_{0}}^{y_{0}} \int_{-x_{0}}^{x_{0}} w\left(x_{r}, x_{0}\right) w\left(y_{r}, y_{0}\right) \boldsymbol{E}_{\boldsymbol{s}}\left(\boldsymbol{r}_{\boldsymbol{r}}, \omega\right) e^{-i\left[k_{x} x_{r}+k_{y} y_{r}\right]} \mathrm{d} x_{r} \mathrm{~d} y_{r}
$$

where $w$ is the Blackman-Harris window given by

$$
w\left(x, x_{0}\right)=a_{0}-a_{1} \cos \left(\pi \frac{x+x_{0}}{x_{0}}\right)+a_{2} \cos \left(2 \pi \frac{x+x_{0}}{x_{0}}\right)-a_{3} \cos \left(3 \pi \frac{x+x_{0}}{x_{0}}\right)
$$

with $a_{0}=0.35875, a_{1}=0.48829, a_{2}=0.14128$, and $a_{3}=0.01168$.

The last approach, which has been investigated for compensating for the finite-sized scan plane, is the use of an asymptotic expansion of the semi-infinite part of the Fourier integral in which the scattered field is not measured. By making a far-field approximation of the forward model in [4], the following expression has been found for the scattered field

$$
\boldsymbol{E}_{\boldsymbol{s}}\left(\boldsymbol{r}_{\boldsymbol{r}}, \omega\right)=e^{2 i k_{0} R_{\mathrm{c}}} \boldsymbol{E}_{\boldsymbol{s}}^{\mathbf{0}}\left(\boldsymbol{r}_{\boldsymbol{r}}, \omega\right) .
$$

Herein, $k_{0}$ is the free-space wavenumber and $R_{c}$ is the distance from the receiving antenna to the center of the scattering object. The function $\boldsymbol{E}_{\boldsymbol{s}}^{\mathbf{0}}$ is slowly varying compared to the exponential function and the integration over the area outside the scan plane can therefore be evaluated using an asymptotic expansion. Applying this approach, the following expression is found for the Fourier transform of the scattered field 


$$
\begin{aligned}
\widetilde{\boldsymbol{E}_{\boldsymbol{s}}^{\text {A.E. }}}\left(k_{x}, k_{y}, \omega\right)= & \int_{-y_{0}}^{y_{0}} \int_{-x_{0}}^{x_{0}} \boldsymbol{E}_{\boldsymbol{s}}\left(\boldsymbol{r}_{\boldsymbol{r}}, \omega\right) e^{-i\left[k_{x} x_{r}+k_{y} y_{r}\right]} \mathrm{d} x_{r} \mathrm{~d} y_{r} \\
& +i \int_{0}^{2 \pi} R_{\min }(\phi) \hat{\boldsymbol{p}}_{\boldsymbol{r}} \cdot \boldsymbol{E}_{\boldsymbol{s}}\left(\boldsymbol{r}_{\boldsymbol{r}}^{\min }(\phi), \omega\right) \frac{e^{-i R_{\min }(\phi)\left[k_{x} \cos \phi+k_{y} \sin \phi\right]}}{2 k_{1}-\left(k_{x} \cos \phi+k_{y} \sin \phi\right)} \mathrm{d} \phi .
\end{aligned}
$$

The last integral in (9) is the compensation for the truncated scan plane. The compensation is found as an end-point contribution using partial integration, as described in [5, Ch. $3]$. It has been assumed that the scan plane is electrically large and that the center of the scattering object is positioned under the center of the scan plane. The depth at which the scattering object is buried is assumed to be much smaller than the distance from the center to the edge of the scan plane. The distance from the center of the scan plane to the edge is denoted by $R_{\min }$ and is given as a function of the azimuthal angle $\phi$ of the receiving antenna. The scan plane with these two parameters is seen in Figure 1. The vector $r_{r}^{\min }$ is the position vector of the receiving antenna when this is positioned at the edge of the scan plane.

Observing the integrand in the compensation integral in (9) it is seen that special care should be taken when applying this algorithm as a singularity is present when

$$
2 k_{1}=k_{x} \cos \phi+k_{y} \sin \phi \text {. }
$$

This singularity is caused by the fact that as the value of $k_{x} \cos \phi+k_{y} \sin \phi$ exceeds $2 k_{1}$, the type of asymptotic expansion changes from being an end-point contribution to a stationarypoint contribution.

\section{Numerical Investigations}

To investigate the impact of using the three different approaches for truncation, a 2.5dimensional setup, as the one used in [4], is considered. Herein, an infinitely long cylinder is buried with its axis parallel to the $x$ axis and the scan is performed along the $y$ axis with an offset in the $\hat{\boldsymbol{y}}$ direction of $0.01 \mathrm{~m}$. The cylinder has a radius of $0.1 \lambda$ and is buried with its axis at $z_{c}=-2 \lambda$. Both antennas are $\hat{\boldsymbol{x}}$ directed and the amplitude of the $\hat{\boldsymbol{x}}$ component of the scattered field $E_{s}^{x}$ as a function of the position of the receiving antenna is seen in Figure 2.

In Figure 3, the amplitudes of the Fourier transforms calculated using the three different truncation techniques are plotted. In each case, the value of $y_{0}$ has been chosen to $2 \lambda$, yielding a total length of the scan line of $4 \lambda$. In addition to the transforms calculated using truncation, a reference transform found by including the scattered field from $y_{r}=-2000 \lambda$ to $y_{r}=2000 \lambda$ is shown. By comparing the results of the transforms calculated using the truncated signal with the reference transform, it is seen that the transform calculated using the Blackman-Harris window yields the worst results while the transform calculated by adding the asymptotic end-point contribution is the best for the lower values of $\left|k_{y}\right|$. For the higher values of $\left|k_{y}\right|$, however, the singularity of the asymptotic expansion implies that the accuracy of the transform calculated using a simple rectangular window yields the best results, although it is influenced by aliasing. In Figure 4 the absolute error in phase when comparing the truncated transforms with the reference is plotted. The conclusions on this plot are similar to those of the amplitude plot: The truncation using the Blackman-Harris window yields the worst results while the compensation using the asymptotic expansion yields good results for those values of $k_{y}$ for which the singularity of the expansion has no effect on the results. 


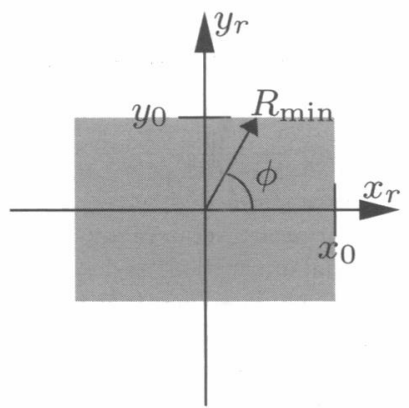

Figure 1: Scan plane and parameters $R_{\min }$ and $\phi$.

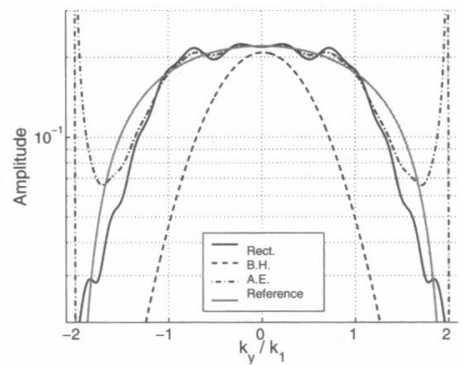

Figure 3: Amplitude of the Fourier transforms calculated using the three different approaches.

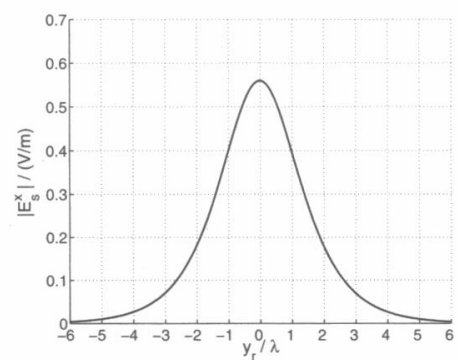

Figure 2: Amplitude of scattered field as function of position of receiving antenna.

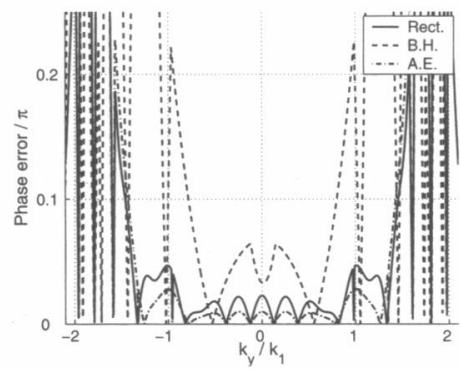

Figure 4: Absolute error in phase of Fourier transforms calculated using the three different approaches.

\section{References}

[1] A. J. Devaney, "A filtered backpropagation algorithm for diffraction tomography," Ultrasonic Imaging, no. 4, pp. 336-350, 1982.

[2] - "Geophysical diffraction tomography," IEEE Trans. Geosci. Remote Sensing, vol. 22, no. 1, pp. 3-13, Jan. 1984.

[3] F. J. Harris, "On the use of windows for harmonic analysis with the discrete Fourier transform," Proc. IEEE, vol. 66, pp. 51-83, 1978.

[4] T. B. Hansen and P. M. Johansen, "Inversion scheme for ground penetrating radar that takes into account the planar air-soil interface," IEEE Transactions on Geoscience and Remote Sensing, vol. 38, no. 1, pp. 496-506, January 2000.

[5] N. Bleistein and R. A. Handelsman, Asymptotic Expansions of Integrals, ser. Dover Books on Advanced Mathematics. New York: Dover Publications, Inc., 1986. 